\title{
ON $L_{W}^{2}$-QUASI-DERIVATIVES FOR SOLUTIONS OF PERTURBED GENERAL QUASI-DIFFERENTIAL EQUATIONS
}

\author{
SOBHY EL-SAYED IBRAHIM
}

\begin{abstract}
This paper is concerned with square integrable quasi-derivatives for any solution of a general quasi-differential equations of $n$th order with complex coefficients $M[y]-\lambda w y=$ $w f\left(t, y^{[0]}, \ldots, y^{[n-1]}\right), t \in[a, b)$ provided that all $r$ th quasi-derivatives of solutions of $M[y]-$ $\lambda w y=0$ and all solutions of its formal adjoint $M^{+}[z]-\lambda w z=0$ are in $L_{w}^{2}(a, b)$ and under suitable conditions on the function $f$.
\end{abstract}

\section{Introduction}

In [16] Anton Zettl proved, under suitable conditions on $f$, that $y^{(j)} \in L^{2}[0, \infty), j=$ $0,1, \ldots, n-1$ for any solution $y$ of $M[y]=f(t, y)$ provided that all $j$ th derivatives of solutions of $M[y]=0$ and all solutions of $M^{+}[y]=0$ are in $L^{2}[0, \infty)$, when $M$ be a regular ordinary linear differential operator of order $n$ with coefficients which are locally integrable on $[0, \infty)$ and $M^{+}$is the formal adjoint of $M$. The case $j=0$ was considered in [16] for general $n$th order $M$ and for $n=2$ by Bradley [1]. Also, in [14] Wong proved that all solutions of a perturbed linear differential equation belong to $L^{2}[0, \infty)$ assuming the fact that all solutions of the unperturbed equation possess the same property, these results generalized by Ibrahim in [10] for general ordinary quasi-differential equations of $n$th order.

Our objective in this paper is to extend the results of Ibrahim, wong and Zettl in [10], [14], [15] and [16] for general ordinary quasi-differential expression $M$ of order $n$ with complex coefficients and to prove under suitable conditions on $f$, that the quasiderivatives $y^{[r]} \in L_{w}^{2}(a, b), r=0,1, \ldots, n-1$ for any solution $y$ of

$$
M[y]-\lambda w y=w f\left(t, y^{[0]}, \ldots, y^{[n-1]}\right)(\lambda \in \mathbb{C}) \text { on }[a, b),
$$

provided that all $r$ th quasi-derivatives of solutions of the equation

$$
M[y]-\lambda w y=0
$$

Received Septembar, 18, 1997.

1991 Mathematics Subject Classification. Primary 34A05, 34B25, 34C11, 34E10, 34E15, 34G10, $47 \mathrm{~A} 55$ and $47 \mathrm{E} 05$.

Key words and phrases. Quasi-differential operators, regular, singular, bounded and square integrable solutions. 
and all solutions of

$$
M^{+}[y]-\bar{\lambda} w y=0
$$

are in $L_{w}^{2}(a, b)$. These results are extensions of those proved by Ibrahim in [10]. Our approach is based on an extension of Gronwall's inequality used by Bradley and due to Gollwitzer [6], on a technical lemma from Goldberg's book [5] and on an appropriate formulation of the variation of parameters formula.

We deal throughout with a quasi-differential expression $M$ of arbitrary order $n$ defined by a Shin-Zettl matrix on the interval $I=(a, b)$. The left-hand end-point of $I$ is assumed to be regular but the right-hand end-point may be either regular or singular.

\section{Notation and Preliminaries}

The set $Z_{n}(I)$ of Shin-Zettl matrices on the interval $I$ consists of $n \times n$ - matrices $A=\left\{a_{r s}\right\}$ whose entries are complex-valued functions on $I$ which satisfy the following conditions:

$$
\left\{\begin{array}{cc}
a_{r s} \in L_{\text {loc }}^{1}(I) & (1 \leq r, s \leq n, n \geq 2) \\
a_{r, r+1} \neq 0 & \text { a.e. on } I \quad(1 \leq r \leq n-1) \\
a_{r s}=0 & \text { a.e. on } I(2 \leq r+1<s \leq n)
\end{array}\right.
$$

For $A \in Z_{n}(I)$, the quasi-derivatives associated with $A$ are defined by

$$
\left\{\begin{array}{l}
y^{[0]}:=y \\
y^{[r]}=a_{r, r+1}^{-1}\left\{\left(y^{[r-1]}\right)^{\prime}-\sum_{s=1}^{r} a_{r s} y^{[s-1]}\right\}, \quad(1 \leq r \leq n-1) \\
y^{[n]}:=\left(y^{[n-1]}\right)^{\prime}-\sum_{s=1}^{n} a_{n s} y^{[s-1]}
\end{array}\right.
$$

where the prime' denotes differentiation.

The quasi-differential expression $M$ associated with $A$ is given by

$$
M[y]=i^{n} y^{[n]}, \quad(n \geq 2)
$$

this being defined on the set

$$
V(M):=\left\{y: y^{[r-1]} \in A C_{l o c}(I), \quad r=1,2, \ldots, n\right\},
$$

where $A C_{l o c}(I)$ denotes the set of all functions which are absolutely continuous on every compact subinterval of $I$.

The formal adjoint $M^{+}$of $\dot{M}$ is defined by the matrix $A^{+} \in Z_{n}(I)$ given by,

$$
A^{+}=-L^{-1} A^{*} L
$$

where $A^{*}$ is the conjugate transpose of $A$ and $L$ is the non-singular $n \times n$ - matrix,

$$
L:=\left\{(-1)^{r} \delta_{r, n+1-s},\right\} \quad(1 \leq r, s \leq n),
$$


$\delta$ being the kronecker delta. If $A^{+}=\left\{a_{r s}^{+}\right\}$, then it follows that

$$
a_{r s}^{+}=(-1)^{r+s+1} \bar{a}_{n-s+1, n-r+1^{\prime}} \text { for each } r \text { and } s .
$$

The quasi-derivatives associated with $A^{+}$are therefore.

$$
\left\{\begin{array}{l}
y_{+}^{[0]}:=y \\
y_{+}^{[r]}:=(\bar{a})_{n-r}^{-1} n={ }^{n-r+1}\left\{\left(y_{+}^{[r-1]}\right)^{\prime}-\sum_{s=1}^{r}(-1)^{r+s+1} \bar{a}_{n-s+1, n-r+1} y_{+}^{[s-1]},\right\}(1 \leq r \leq n-1)(2.8 \\
y_{+}^{[n]}:=\left(y_{+}^{[n-1]}\right)^{\prime}-\sum_{s=1}^{n}(-1)^{n+s+1} \bar{a}_{n-s+1,1} y_{+}^{[s-1]}
\end{array}\right.
$$

and

$$
M^{+}[y]=i^{n} y_{+}^{[n]} \quad(n \geq 2)
$$

for all $y$ in

$$
V\left(M^{+}\right):=\left\{y: y_{+}^{[r-1]} \in A C_{l o c}(I), r=1,2, \ldots, n\right\} .
$$

Note that $\left(A^{+}\right)^{+}=A$ and so $\left(M^{+}\right)^{+}=M$. We refere to [3], [9] and [17] for a full account of the above and subsequent result on quasi-differential expressions.

Let the interval $I$ have end-points $a$ and $b,-\infty \leq a<b \leq \infty$, and let $w$ be a function which satisfies,

$$
w>0 \text { almost everywhere on } I, \quad w \in L_{l o c}^{1}(I) .
$$

The equation

$$
M[y]=\lambda w y, \quad(\lambda \in \mathbb{C})
$$

on $I$ is said to be regular at the left end-point a if for all $x \in(a, b)$,

$$
a \in \mathbb{R}, a_{r s} \in L^{1}[a, x], \quad(r, s=1,2, \ldots, n) .
$$

Otherwise (2.12) is said to be singular at $a$. Similarly we define the terms regular and singular at b. If (2.12) is regular at both end-points, then it is said to be regular; in this case we have

$$
a, b \in \mathbb{R}, a_{r s} \in L^{1}(a, b),(r, s=1,2, \ldots, n) ;
$$

see [2], [8] and [9].

We shall be concerned with the case when a is a regular end-point for (2.12), but the end-point $b$ being allowed to be either regular or singular. Note that in view of (2.7), an end-point of the interval $I$ is regular for $(2,12)$ if and only if it is regular for the equation,

$$
M^{+}[y]=\bar{\lambda} w y \quad(\lambda \in \mathbb{C})
$$

Let $L_{w}^{2}(a, b)$ denote the usual weighted $L^{2}$-space with inner-product

$$
(f, g)=\int_{a}^{b} f(x) \overline{g(x)} w(x) d x
$$

and norm $\|f\|:=(f, f)^{1 / 2}$, this is a Hilbert space on identfying functions which differ only on null space. 
Denote by $S(M)$ and $S\left(M^{+}\right)$the sets of all solutions of $M[y]-\lambda w y=0$ and $M^{+}[y]-$ $\bar{\lambda} w y=0$, respectively, and $S^{r}(M)=\left\{y^{[r]} \mid M[y]-\lambda w y=0, r=0,1, \ldots, n-1\right\}$ denotes the set of all quasi-derivatives of solutions of $M[y]-\lambda w y=0$, etc. Let $\phi_{k}(t, \lambda)$ for $k=1,2, \ldots, n$ be the solutions of the homogeneous equation (1.2) determined by the initial conditions,

$$
\phi_{k}^{[r]}\left(t_{0}, \lambda\right)=\delta_{k, r+1^{\prime}} \quad \text { for all } t_{0} \in[a, b),
$$

$(k=1,2, \ldots, n ; r=0,1, \ldots, n-1)$. Then $\phi_{k}^{[r]}\left(t_{0}, \lambda\right)$ is continuous in $(t, \lambda)$ for $a<t<b$, $|\lambda|<\infty$, and for fixed $t$ it is entire in $\lambda$. Let $\phi_{k}^{+}(t, \lambda)$ for $k=1,2, \ldots, n$ be the solutions of the homogeneous equation (1.3) determined by the initial conditions,

$$
\left(\phi_{k}^{+}\right)^{[r]}\left(t_{0}\right)=(-1)^{k+r} \delta_{k, n-r^{\prime}}, \text { for all } t_{0} \in[a, b),
$$

$(k=1,2, \ldots, n ; r=0,1, \ldots, n-1)$.

Suppose $a<t_{0}<b$. According to Gilbert [4, Section 3] and Ibrahim [11, Section 3], a solution of $M[\phi]-\lambda w \phi=w f, f \in L_{w}^{1}(a, b)$ satisfying $\phi^{[r]}\left(t_{0}, \lambda\right)=\alpha_{r+1}, r=$ $0,1, \ldots, n-1$, is given by

$$
\begin{aligned}
& \quad \phi(t, \lambda)=\sum_{j=1}^{n} \alpha_{j}(\lambda) \phi_{j}\left(t, \lambda_{0}\right)+\left[\left(\lambda-\lambda_{0}\right) /\left(i^{n}\right)\right] \sum_{j, k=1}^{n} \xi^{j k} \phi_{j}\left(t, \lambda_{0}\right) \\
& \cdot \int_{\dot{t}_{0}}^{t} \phi_{j}^{+}\left(s, \lambda_{0}\right) f(s) w(s) d s
\end{aligned}
$$

where $\phi_{k}^{+}(t, \lambda)$ stands for the complex conjugate of $\phi_{k}(t, \lambda)$, and for each $j, k, \xi^{j k}$ is a constant which is independent of $t, \lambda$ (but does depend in general on $t$ ), also see [9, Corollary 3.10] and [17, Theorem 3].

Theorem 2.1. (Existence and Uniqueness Theorem). Suppose $f \in L_{w}^{1}(a, b)$ and suppose that the conditions (2.1) are satisfied. Then given any complex numbers $\alpha_{j} \in$ $\mathbb{C}, j=0, \ldots, n-1$ and $t_{0} \in(a, b)$ there exists a unique solution of $M[\phi]-\lambda \phi w=w f$ in $(a, b)$ which satisfies

$$
\phi^{[r]}\left(t_{0}, \lambda\right)=\alpha_{r+1}, \quad r=0, \ldots, n-1 .
$$

Proof. See [2, Theorem 3.10.1], [8, Theorem 16.2.2] and [9, Theorem 1.11].

\section{Some Technical Lemmas}

Our first lemma is a form of the variation of parameters formula. However it is different from the form of this formula generally found in textbooks and the literature. For the variation of parameters formula for general quasi-differential equations, see [11, Section 3] and [17, Theorem 3]. These contain Lemma 3.1 as a special case, see [16]. 
Lemma 3.1. Suppose $f$ is locally $L_{w}^{1}(a, b)$ function and $\phi(t, \lambda)$ is the solution of $M[y]-\lambda w y=w f$ satisfying

$$
\phi^{[r]}\left(t_{0}, \lambda\right)=\alpha_{r+1}(\lambda), \quad t_{0} \in[a, b) \quad \text { for all } r=0,1, \ldots, n-1 .
$$

Then,

$$
\begin{aligned}
\phi^{[r]}(t, \lambda)= & \sum_{j=1}^{n} \alpha_{j}(\lambda) \phi_{j}^{[r]}\left(t, \lambda_{0}\right)+\left[\left(\lambda-\lambda_{0}\right) /\left(i^{n}\right)\right] \sum_{j, k=1}^{n} \xi^{j k} \phi_{j}^{[r]}\left(t, \lambda_{0}\right) \\
& \cdot \int_{a}^{t} \overline{\phi_{j}^{+}\left(s, \lambda_{0}\right)} f(s) w(s) d s \quad \text { for } r=0,1, \ldots, n-1 .
\end{aligned}
$$

Crucial in the study of boundedness of solutions of quasi-differential equations is the fundamental inequality of Gronwall, see [7]. Here, we shall also need the following variant which may be found in [13].

Lemma 3.2. Let $u(t), v(t)$ be two non-negative functions, locally integrable on $[a, b)$. Then, the following inequality for $0 \leq p<1$,

$$
u(t) \leq C_{0}+\int_{a}^{t} v(s) u^{p}(s) d s, \quad C_{0}>0
$$

implies that,

$$
u(t) \leq\left[C_{0}^{1-p}+(1-p) \int_{a}^{t} v(s) d s\right]^{1 /(1-p)}
$$

In particular, if $v(t) \in L^{1}(a, b)$, then (3.2) implies that $u(t)$ is bounded.

The next lemma is a special case of an extension of the well-known Gronwall inequality due to Gollwitzer [6] (See also Willett [12] and Willet-Wong [13]).

Lemma 3.3. Let $u, z, g, h$, be continuous non-negative functios on $[a, b)$ and suppose that

$$
u(t) \leq z(t)+g(t)\left[\int_{a}^{t} u^{2}(s) h(s) d s\right]^{\frac{1}{2}} \quad \text { for } t \geq a .
$$

Then,

$$
u(t) \leq z(t)+g(t)\left[\int_{a}^{t} 2 z^{2}(s) h(s) \exp \left[\int_{a}^{s} 2 g^{2}(x) h(x) d x\right] d s\right)^{\frac{1}{2}} \text { for } t \geq a .
$$

The next two Lemmas has been proved in [9] for general case.

Lemma 3.4. ([9, Proposition 3.23]) If all solutions of $M[\phi]=\lambda_{0} w \phi$ are bounded on $[a, b)$ for some $\lambda_{0} \in \mathbb{C}$. and $\phi_{j}^{+} \in L_{w}^{1}(a, b)$ for $j=1,2, \ldots, n$, then all solutions of $M[\phi]=\lambda w \phi$ are also bounded on $[a, b)$ for all $\lambda \in \mathbb{C}$.

Lemma 3.5. ([9, Proposition 3.24]) If all solutions of $M[\phi]=\lambda_{0} w \phi$ and $M^{+}[\phi]=$ $\bar{\lambda}_{0} w \phi$ are in $L_{w}^{2}(a, b)$ for some $\lambda_{0} \in \mathbb{C}$, then all solutions of $M[\phi]=\lambda w \phi$ and $M^{+}[\phi]=$ $\bar{\lambda} w \phi$ are in $L_{w}^{2}(a, b)$ for all $\lambda \in \mathbb{C}$. 


\section{The Main Results}

Suppose there exist non-negative continuous functions $k(t)$ and $h_{i}(t), i=0, \ldots, n-1$ such that

$$
\left|f\left(t, y^{[0]}, \ldots, y^{[n-1]}\right)\right| \leq k(t)+\sum_{i=0}^{n-1} h_{i}(t)\left|y^{[i]}\right|^{\sigma},
$$

for $t \geq a,-\infty<y^{[i]}<\infty$, for each $i=0, \ldots, n-1 ; 0 \leq \sigma \leq 1$.

Theorem 4.1. Suppose $f$ satisfies (4.1) with $\sigma=1, S^{r}(M) \cup S\left(M^{+}\right) \subset L^{\infty}(a, b)$ for some $r=0,1, \ldots, n-1$ and some $\lambda_{0} \in \mathbb{C}$ and that,

(i) $k(t) \in L_{w}^{1}(a, b)$ for all $t \in[a, b)$,

(ii) $h_{i}(t) \in L_{w}^{1}(a, b)$ for all $t \in[a, b), i=0,1, \ldots, n-1$.

Then $\phi^{[r]}(t, \lambda)$ is bounded on $[a, b)$ for any solution $\phi(t, \lambda)$ of the equation (1.1) for all $\lambda \in \mathbb{C}$.

Proof. Note that (4.1) and Theorem 2.1 implies that all solutions exist on the entire interval $[a, b)$, see [2, Chapter 3], [10], [14] and [15].

Let $\left\{\phi_{1}\left(t, \lambda_{0}\right), \ldots, \phi_{n}\left(t, \lambda_{0}\right)\right\}$ and $\left\{\phi_{1}^{+}\left(t, \lambda_{0}\right), \ldots, \phi_{n}^{+}\left(t, \lambda_{0}\right)\right\}$ be two sets of linearly independent solutions of (1.2) and (1.3) respectively and let $\phi(t, \lambda)$ be any solution of (1.1) on $[a, b)$, then by Lemma 3.1, we have,

$$
\begin{aligned}
\phi^{[r]}(t, \lambda)= & \sum_{j=1}^{n} \alpha_{j}(\lambda) \phi_{j}^{[r]}\left(t, \lambda_{0}\right)+\left(\left(\lambda-\lambda_{0}\right) /\left(i^{n}\right)\right) \sum_{j, k=1}^{n} \xi^{j k} \phi_{j}^{[r]}\left(t, \lambda_{0}\right) \\
& \cdot \int_{a}^{t} \overline{\phi_{j}^{+}\left(s, \lambda_{0}\right)} f\left(s, \phi^{[0]}, \ldots, \phi^{[n-1]}\right) w(s) d s, \quad \text { for } r=0, \ldots, n-1 .
\end{aligned}
$$

Hence,

$$
\begin{aligned}
\left|\phi^{[r]}(t, \lambda)\right| \leq & \sum_{j=1}^{n}\left|\alpha_{j}(\lambda)\right|\left|\phi_{j}^{[r]}\left(t, \lambda_{0}\right)\right|+\left|\lambda-\lambda_{0}\right| \sum_{j, k=1}^{n}\left|\xi^{j k}\right|\left|\phi_{j}^{[r]}\left(t, \lambda_{0}\right)\right| \\
& \cdot \int_{a}^{t} \overline{\left|\phi_{k}^{+}\left(s, \lambda_{0}\right)\right|}\left(k(s)+\sum_{i=0}^{n-1} h_{i}(s)\left|\phi^{[i]}(s, \lambda)\right|\right) w(s) d s, r=0,1, \ldots, n-1 .
\end{aligned}
$$

Since, $k(t) \in L_{w}^{1}(a, b)$ and $\phi_{j}^{+}\left(t, \lambda_{0}\right)$ bounded on $[a, b)$ for some $\lambda_{0} \in \mathbb{C}$, then $\phi_{j}^{+} k \in$ $L_{w}^{1}(a, b), j=1,2, \ldots, n$ for some $\lambda_{0} \in \mathbb{C}$.

Setting,

$$
C_{j}=\left|\lambda-\lambda_{0}\right| \sum_{j, k=1}^{n}\left|\xi^{j k}\right| \int_{a}^{b} \overline{\mid \phi_{j}^{+}\left(s, \lambda_{0}\right)} \mid k(s) w(s) d s, \quad(j=1,2, \ldots, n),
$$

then,

$$
\left|\phi^{[r]}(t, \lambda)\right| \leq \sum_{j=1}^{n}\left(c_{j}+\left|\alpha_{j}(\lambda)\right|\right) \phi_{j}^{[r]}\left(t, \lambda_{0}\right)|+| \lambda-\lambda_{0}\left|\sum_{j, k=1}^{n} \sum_{i=0}^{n-1}\right| \xi^{j k} \mid
$$




$$
\cdot\left|\phi_{j}^{[r]}\left(t, \lambda_{0}\right)\right| \int_{a}^{t} \overline{\mid \phi_{j}^{+}\left(s, \lambda_{0}\right)}\left|h_{i}(s)\right| \phi^{[i]}(s, \lambda) \mid w(s) d s, r=0,1, \ldots, n-1 .
$$

By hypothesis, there exist positive constants $K_{0}$ and $K_{1}$ such that,

$$
\left|\phi_{j}^{[r]}\left(t, \lambda_{0}\right)\right| \leq K_{0} \text { and }\left|\phi_{j}^{+}\left(t, \lambda_{0}\right)\right| \leq K_{1} \text {, for all } t \in[a, b),
$$

$j=1,2, \ldots, n$ and some $r=0,1, \ldots, n-1$.

Hence, by summing both sides of (4.5) from $r=0, \ldots, n-1$ we get,

$$
\begin{aligned}
\left(\sum_{r=0}^{n-1}\left|\phi^{[r]}(t, \lambda)\right|\right) \leq & (n-1)) K_{0} \sum_{j=1}^{n}\left(C_{j}+\left|\alpha_{j}(\lambda)\right|\right)+(n-1) K_{0} K_{1}\left|\lambda-\lambda_{0}\right| \\
& \cdot \sum_{j, k=1}^{n}\left|\xi^{j k}\right| \int_{a}^{t}\left(\max _{0 \leq i \leq n-1} h_{i}(s)\right)\left(\sum_{i=0}^{n-1}\left|\phi^{[i]}(s, \lambda)\right|\right) w(s) d s .
\end{aligned}
$$

On application of Gronwall's inequality to (4.6) and from (ii), we deduce that $\sum_{r=0}^{n-1}\left|\phi^{[r]}(t, \lambda)\right|$ is finite and hence the result.

Remark. From [16, Section 3] and [9, Lemma 3.3], $\phi$ and $\phi^{[j]} \in L_{w}^{2}(a, b)$ implies that $\phi^{[r]} \in L_{w}^{2}(a, b)$ for any solution $\phi$ of the equation (1.1), for all $r=1, \ldots, j-1$, $1 \leq j \leq n-1$.

Theorem 4.2. Suppose $f$ satisfies (4.1) with $\sigma=1, S^{r}(M) \cup S\left(M^{+}\right) \subset L_{w}^{2}(a, b)$ for some $\lambda_{0} \in \mathbb{C}$ and some $r=0,1, \ldots, n-1$ and that $(i) k(t) \in L_{w}^{2}(a, b)(i i) h_{i}(t) \in$ $L^{\infty}(a, b), i=0,1, \ldots, n-1$, for all $t \in[a, b)$. Then $\phi^{[r]}(t, \lambda) \in L_{w}^{2}(a, b)$ for any solution $\phi(t, \lambda)$ of the equation (1.1) for all $\lambda \in \mathbb{C}$.

Proof. On application of the Cauchy Schwartz inequality to the integral in (4.5) we get,

$$
\begin{aligned}
\left|\phi^{[r]}(t, \lambda)\right| \leq & \sum_{j=1}^{n}\left(C_{j}+\left|\alpha_{j}(\lambda)\right|\right)\left|\phi_{j}^{[r]}\left(t, \lambda_{0}\right)\right|+\left|\lambda-\lambda_{0}\right| \sum_{j, k=1}^{n} \sum_{i=0}^{n-1}\left|\xi^{j k}\right| \\
& \cdot\left|\phi_{j}^{[r]}\left(t, \lambda_{0}\right)\right|\left(\left.\int_{a}^{t} \overline{\mid \phi_{j}^{+}\left(s, \lambda_{0}\right)}\right|^{2}\left|h_{i}(s)\right| w d s\right)^{\frac{1}{2}}\left(\int_{a}^{t}\left|h_{i}(s)\right|\left|\phi^{[i]}(s, \lambda)\right|^{2} w d s\right)^{\frac{1}{2}},
\end{aligned}
$$

$r=0,1, \ldots, n-1$.

Since $\phi_{j}^{+}\left(t, \lambda_{0}\right) \in L_{w}^{2}(a, b), j=1,2, \ldots, n$ for some $\lambda_{0} \in \mathbb{C}$ and $h_{i}(t) \in L^{\infty}(a, b)$ by hypothesis, then $\phi_{j}^{+}\left(t, \lambda_{0}\right)\left|h_{i}(t)\right|^{1 / 2} \in L_{w}^{2}(a, b), j=1,2, \ldots, n, i=0,1, \ldots, n-1$. Let,

$$
D_{j i}=\left(\left.\int_{a}^{t} \overline{\mid \phi_{j}^{+}\left(s, \lambda_{0}\right)}\right|^{2}\left|h_{i}(s)\right| w(s) d s\right)^{\frac{1}{2}}, z(t)=\sum_{j=1}^{n}\left(C_{j}+\left|\alpha_{j}(\lambda)\right|\right)\left|\phi_{j}^{[r]}\left(t, \lambda_{0}\right)\right|,
$$

and $G(t)=\left|\lambda-\lambda_{0}\right| \sum_{j, k=1}^{n} \sum_{i=0}^{n-1} D_{j i}\left|\xi^{j k}\right|\left|\phi_{j}^{[r]}\left(t, \lambda_{0}\right)\right|$. 
From Lemma 3.3 we have,

$$
\left|\phi^{[r]}(t, \lambda)\right| \leq Z(t)+G(t)\left(\int_{a}^{t} 2 Z^{2}(s)\left|h_{i}(s)\right| \exp \left[\int_{a}^{s} 2 G^{2}(x)\left|h_{i}(x)\right| w d x\right] w d s\right)^{\frac{1}{2}} .
$$

Since $\int_{a}^{b} Z^{2}(s)\left|h_{i}(s)\right| w(s) d s$ and $\int_{a}^{b} G^{2}(x)\left|h_{i}(x)\right| w(x) d x$ are both finite, we concelude that $\left|\phi^{[r]}(t, \lambda)\right|$ is bounded by a linear combination of the $L_{w}^{2}(a, b)$ functions $Z(t)$ and $G(t)$. Therefore, by using Lemma $3.5, \phi^{[r]}(t, \lambda) \in L_{w}^{2}(a, b)$ for all $\lambda \in \mathbb{C}$.

Remark. If we use the Cauchy Schwartz inequality to the integral in (4.5) as:

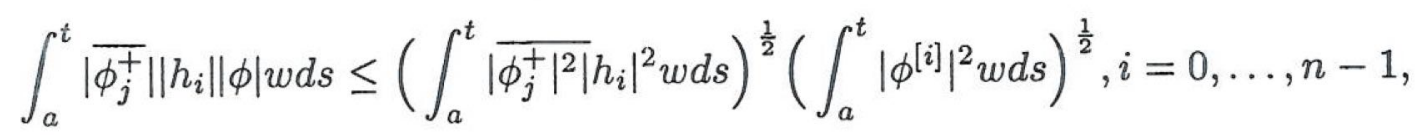

we get also the result. We refer to [14] and [15] for more details.

Corollary 4.3. Suppose that $f\left(t, y^{[0]}, \ldots, y^{[n-1]}\right)=\sum_{i=1}^{n-1} h_{i}(t) y^{[i]}, S^{r}(M) \cup S\left(M^{+}\right) \subset$ $L_{w}^{2}(a, b)$ for some $\lambda_{0} \in \mathbb{C}$ and some $r=0,1, \ldots, n-1$ and that $h_{i}(t) \in L_{w}^{p}(a, b)$ for some $p \geq 2, t \in[a, b) ; i=0,1, \ldots, n-1$. Then $\phi^{[r]}(t, \lambda) \in L_{w}^{1}(a, b)$ for any solution $\phi(t, \lambda)$ of the equation (1.1) for all $\lambda \in \mathbb{C}$ and all $r=0,1, \ldots, n-1$.

Proof. The proof is similar to Theorem 4.2 and therefore omitted.

The special case $h_{i}(t) \equiv 0, i=0, \ldots, n-1$ and $k \in L_{w}^{2}(a, b)$ yields:

Corollary 4.4. If all solutions of $M[\phi]=\lambda_{0} w \phi$ and $M^{+}[\phi]=\bar{\lambda}_{0} w \phi$ are in $\in L_{w}^{2}(a, b)$ for some $\lambda_{0} \in \mathbb{C}$ and $k \in L_{w}^{2}(a, b)$, then all solutions of $M[\phi]-\lambda w \phi=w k$ are in $L_{w}^{2}(a, b)$ for all $\lambda \in \mathbb{C}$.

Next, we consider (4.1) with $0 \leq \sigma<1$, and have the following:

Theorem 4.5. Suppose $f$ satisfies (4.1) with $0 \leq \sigma<1, S^{r}(M) \cup S\left(M^{+}\right) \subset L_{w}^{2}(a, b)$ for some $\lambda_{0} \in \mathbb{C}$ and some $r=0,1, \ldots, n-1$ and that,

(i) $k(t) \in L_{w}^{2}(a, b)$ for all $t \in[a, b)$,

(ii) $h_{i}(t) \in L_{w}^{2 /(1-\sigma)}(a, b) 0 \leq \sigma<1, i=0,1, \ldots, n-1$.

Then $\phi^{[r]}(t, \lambda) \in L_{w}^{2}(a, b)$ for any solution $\phi(t, \lambda)$ of the equation (1.1) for all $\lambda \in \mathbb{C}$.

Proof. For $0 \leq \sigma<1$, the proof is the same up to (4.5). In this case (4.5) becomes,

$$
\begin{aligned}
\left|\phi^{[r]}(t, \lambda)\right| \leq & \sum_{j=1}^{n}\left(c_{j}+\left|\dot{\alpha}_{j}(\lambda)\right|\right)\left|\phi_{j}^{[r]}\left(t, \lambda_{0}\right)\right|+\left|\lambda-\lambda_{0}\right| \sum_{j, k=1}^{n} \sum_{i=0}^{n-1}\left|\xi^{j k}\right| \\
& \left.\cdot\left|\phi_{j}^{[r]}\left(t, \lambda_{0}\right)\right| \int_{a}^{t} \overline{\mid \phi_{j}^{+}\left(s, \lambda_{0}\right)}\left|h_{i}(s)\right| \phi^{[i]}(s, \lambda)\right|^{\sigma} w(s) d s, r=0,1, \ldots, n-1 .(4 .
\end{aligned}
$$

Applying Cauchy Schwartz inequality to the integral in (4.8), we find

$$
\begin{aligned}
& \left.\int_{a}^{t} \overline{\mid \phi_{j}^{+}\left(s, \lambda_{0}\right)}\left|h_{i}(s)\right| \phi(s, \lambda)\right|^{\sigma} w(s) d s \\
& \quad \leq\left(\left.\int_{a}^{t} \overline{\mid \phi_{j}^{+}\left(s, \lambda_{0}\right)} h_{i}(s)\right|^{\mu} w(s) d s\right)^{\frac{1}{\mu}}\left(\int_{a}^{t}\left|\phi^{[i]}(s, \lambda)\right|^{2} w(s) d s\right)^{\frac{\sigma}{2}}
\end{aligned}
$$


where $\mu=2 /(2-\sigma)$. Since $\phi_{j}^{+}\left(s, \lambda_{0}\right) \in L_{w}^{2}(a, b)$ for some $\lambda_{0} \in \mathbb{C}, j=1,2, \ldots, n$ and $h_{i}(s) \in L_{w}^{2 /(1-\sigma)}(a, b)$ by hypothesis, then we have $\phi_{j}^{+}\left(s, \lambda_{0}\right) h_{i}(s) \in L_{w}^{\mu}(a, b)$ for some $\lambda_{0} \in \mathbb{C}, j=1,2, \ldots, n ; i=0,1 \ldots, n-1$. Using this fact and (4.9) in (4,8), we obtain

$$
\begin{aligned}
\left|\phi^{[r]}(t, \lambda)\right| \leq & \sum_{j=1}^{n}\left(c_{j}+\left|\alpha_{j}(\lambda)\right|\right)\left|\phi_{j}^{[r]}\left(t, \lambda_{0}\right)\right|+K_{0}\left|\lambda-\lambda_{0}\right| \sum_{j, k=1}^{n} \sum_{i=0}^{n-1}\left|\xi^{j k}\right| \\
& \cdot\left|\phi_{j}^{[r]}\left(t, \lambda_{0}\right)\right|\left(\int_{a}^{t}\left|\phi^{[i]}(s, \lambda)\right|^{2} w(s) d s\right)^{\frac{\sigma}{2}}, r=0,1, \ldots, n-1,
\end{aligned}
$$

where $K_{0}=\left\|\phi_{j}^{+}\left(t, \lambda_{0}\right) h_{i}(s)\right\|_{\mu},\|\cdot\|_{\mu}$ denotes the norm in $L_{w}^{\mu}(a, b)$. From the inequality

$$
(u+v)^{2} \leq 2\left(u^{2}+v^{2}\right)
$$

it follows that

$$
\begin{aligned}
&\left|\phi^{[r]}(t, \lambda)\right|^{2} \leq 4 \sum_{j=1}^{n}\left(c_{j}^{2}+\left|\alpha_{j}(\lambda)\right|^{2}\right)\left|\phi_{j}^{[r]}\left(t, \lambda_{0}\right)\right|^{2}+4 K_{0}^{2}\left|\lambda-\lambda_{0}\right|^{2} \sum_{j, k=1}^{n} \sum_{i=0}^{n-1}\left|\xi^{j k}\right|^{2} \\
& \cdot\left|\phi_{j}^{[r]}\left(t, \lambda_{0}\right)\right|^{2}\left(\int_{a}^{t}\left|\phi^{[i]}(s, \lambda)\right|^{2} w(s) d s\right)^{\sigma}, \quad r=0,1, \ldots, n-1 .
\end{aligned}
$$

Setting, $K_{1}=\int_{a}^{b}\left|\phi_{j}^{[r]}\left(t, \lambda_{0}\right)\right|^{2} w(s) d s$, for some $\lambda_{0} \in \mathbb{C}$ and some $r=0, \ldots, n-1 ; j=$ $1,2, \ldots, n$ and integrate (4.12) to obtain

$$
\begin{aligned}
\int_{a}^{t}\left|\phi^{[r]}(s, \lambda)\right|^{2} w(s) d s \leq & K_{2}+4 K_{0}^{2}\left|\lambda-\lambda_{0}\right|^{2}\left(\sum_{j, k=1}^{n} \sum_{i=0}^{n-1}\left|\xi^{j k}\right|^{2} \int_{a}^{t}\left|\phi_{j}^{[r]}\left(s, \lambda_{0}\right)\right|^{2}\right. \\
& \cdot\left[\left(\int_{a}^{s}\left|\phi^{[i]}(x, \lambda)\right|^{2} w(x) d x\right)^{\sigma}\right] w(s) d s
\end{aligned}
$$

where

$$
K_{2}=4 \sum_{j=1}^{n}\left(c_{j}^{2}+\left|\alpha_{j}(\lambda)\right|^{2}\right) K_{1}
$$

On application of Lemma 3.2 to (4.13) for $0 \leq \sigma<1$ and of Gronwall's inequality to (4.13) for $\sigma=1$, yields the result.

Theorem 4.6. Suppose $f$ satisfies (4.1) with $0 \leq \sigma<1, S^{r}(M) \cup S\left(M^{+}\right) \subset L_{w}^{2}(a, b) \cap$ $L^{\infty}(a, \dot{b})$ for some $\lambda_{0} \in \mathbb{C}$ and some $r=0,1, \ldots, n-1$ and that,

(i) $k(t) \in L_{w}^{2}(a, b)$ for all $t \in[a, b)$,

(ii) $h_{i}(t) \in L_{w}^{p}(a, b)$ for any $p, 1 \leq p \leq 2 /(1-\sigma), i=0,1, \ldots, n-1$. Then $\phi^{[r]}(t, \lambda) \in L_{w}^{2}(a, b) \cap L^{\infty}(a, b)$ for any solution $\phi(t, \lambda)$ of the equation (1.1) for all $\lambda \in \mathbb{C}$. 
Proof. Since $S^{r}(M) \cup S\left(M^{+}\right) \subset L_{w}^{2}(a, b) \cap L^{\infty}(a, b)$ for some $\lambda_{0} \in \mathbb{C}$ and some $r=0,1, \ldots, n-1$, then $\phi_{j}^{[r]}\left(t, \lambda_{0}\right), \phi_{j}^{+}\left(t, \lambda_{0}\right) \in L_{w}^{q}(a, b), j=1,2, \ldots, n$ for every $q \geq 2$ and for some $\lambda_{0} \in \mathbb{C}, r=0,1, \ldots, n-1$.

First, suppose that $h_{i}(t) \in L_{w}^{p}(a, b)$ for some $p, 1 \leq p \leq 2$. Setting,

$$
K_{0}=\left\|\phi^{[r]}\left(t, \lambda_{0}\right)\right\|_{\infty} \text { and } K_{1}=\left\|\phi_{j}^{+}\left(t, \lambda_{0}\right)\right\|_{\infty}, j=1,2, \ldots, n
$$

for some $\lambda_{0} \in \mathbb{C}$ and some $r=0,1, \ldots, n-1$, we have from (4.8),

$$
\begin{aligned}
\left|\phi^{[r]}(t, \lambda)\right| \leq & K_{0}\left(\sum_{j=1}^{n}\left(c_{j}+\left|\alpha_{j}(\lambda)\right|\right)\right)+K_{0} K_{1}\left|\lambda-\lambda_{0}\right|\left(\sum_{j, k=1}^{n} \sum_{i=0}^{n-1}\left|\xi^{j k}\right|\right. \\
& \left.\cdot \int_{a}^{t} h_{i}(s)\left|\phi^{[i]}(s, \lambda)\right|^{\sigma} w(s) d s\right) .
\end{aligned}
$$

Since $h_{i}(t) \in L_{w}^{p}(a, b), 1 \leq p \leq 2$, then Lemma 3.2 together with Gronwall's inequality implies that $\phi^{[r]}(t, \lambda) \in L^{\infty}(a, b)$ for all $\lambda \in \mathbb{C}$, i.e., there exist a positive constant $K_{2}$ such that,

$$
\left|\phi^{[r]}(t, \lambda)\right| \leq K_{2} \text { for all } \lambda \in \mathbb{C}, t \in[a, b), r=0,1, \ldots, n-1
$$

From (4.8) and (4.15), we obtain

$$
\left|\phi^{[r]}(t, \lambda)\right| \leq \sum_{j=1}^{n}\left(c_{j}+\left|\alpha_{j}(\lambda)\right|+K_{3}\right)\left|\phi_{j}^{[r]}\left(t, \lambda_{0}\right)\right| .
$$

for some appropriate constant $K_{3}$. Since $\phi_{j}^{[r]}\left(t, \lambda_{0}\right) \in L_{w}^{2}(a, b)$ for some $\lambda_{0} \in \mathbb{C}$ and some $r=0,1, \ldots, n-1$, this proves $\phi^{[r]}(t, \lambda) \in L_{w}^{p}(a, b)$ for all $\lambda \in \mathbb{C}, 1 \leq p \leq 2$.

Next, suppose that $h_{i}(t) \in L_{w}^{p}(a, b), 2<p \leq 2 /(1-\sigma) ; i=0,1, \ldots, n-1$. Define $q \geq 2$ by

$$
\frac{1}{q}=\frac{2-\sigma}{2}-\frac{1}{p}
$$

(which is possible because of the restriction on $p$ ). Thus $\phi_{j}^{[r]}\left(t, \lambda_{0}\right) \phi_{j}^{+}\left(t, \lambda_{0}\right) \in L_{w}^{q}(a, b)$ and $\phi_{j}^{+}\left(t, \lambda_{0}\right) h_{i}(t) \in L_{w}^{\mu}(a, b), \mu=2 /(2-\sigma)$.

Repeating the same argument in the proof of Theorem 4.5, from (4.8) to (4.13), we obtain the fact that $\phi^{[r]}(t, \lambda) \in L_{w}^{2}(a, b)$. Returning to (4.9), we find that the integral on the left-hand side is bounded which implies, by (4.8) that,

$$
\left|\phi^{[r]}(t, \lambda)\right| \leq \sum_{j=1}^{n}\left(c_{j}+\left|\alpha_{j}(\lambda)\right|+K_{3}\right)\left|\phi_{j}^{[r]}\left(t, \lambda_{0}\right)\right|
$$

for some appropriate constant $K_{3}$. Since $\phi_{j}^{[r]}\left(t, \lambda_{0}\right) \in L^{\infty}(a, b)$, this completes the proof. We refer to [10], [14] and [16] for more details. 


\section{References}

[1] J. S. Bradley, "Comparison theorems for the square integrability of solutions of $\left(r(t) y^{\prime}\right)^{\prime}+$ $q(t) y=f(t, y), "$ Glasgow Math. Soc., 13 (1972), 75-79.

[2] D. E. Edmunds and W. D. Evans, Spectral Theory and Differential Operators, Oxford University press, 1987.

[3] W. N. Everitt and D. Race, "Some remarks on linear ordinary quasi-differential expressions," Proc. London Math. Soc., (3), 54(1987), 300-320.

[4] R. C. Gilbert, "Simplicity of linear ordinary differential operatiors, " Journal of Differential Equations, 11(1972), 672-681.

[5] S. Goldberg, "Unbounded linear operators," McGraw. Hill, New York, 1966.

[6] H. E. Gollwitzer, "A note on a functional inequality," Proc. Amer. Math. Soc., 23(1969), 642-647.

[7] M. R Mohana Rao, "Ordinary differential equations," Theory and Applications, First published in the United Kingdom in 1989 by Edward Arnold (Publishers) Limited, London.

[8] M. N. Nainark, "Linear differential operators," G. I. T. T. L., Moscow (1954), Ungar, New York, I(1967), II(1968).

[9] Sobhy El-sayed Ibrahim, "Problems associated with differential operators," Ph. D. thesis (1989), Faculty of Science, Department of Mathematics, Benha University, Egypt.

[10] _ Boundedness for solutions of general ordinary quasi-differential equations," Journal of Egyptian Mathematical Society, 2(1994), 33-44.

[11] _ "The spectra of well-posed operators," Proc. Royal Soc. of Edinburgh, 124A(1995), 1331-1348.

[12] D. Willett, "Nonlinear vector integral equations as contraction mappings," Arch. Rational Mech. Anal., 15(1964), 79-86.

[13] D. Willett and J. S. W. Wong, "On the discrete analogues of some generalizations of Gron-

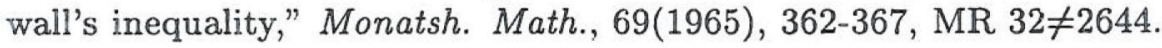

[14] J. S. W. Wong, "Square integrable solutions of perturbed linear differential equations," Proc. Royal Society of Edinburgy, 73A, 16, (1974/75), 251-254.

[15] A. Zettl, "Square integrable solutions of $L y=f(t, y)$," Proceedings of the American Mathematical Society, 26(1970), 635-639.

[16] —- "Perturbation of the limit circle case," Quart. J. Math., Oxford (3), 26(1975), 355-360.

[17] A. Zettl, "Formally self-adjoint quasi-differential operators," Rocky. Mountain Journal of Mathematics, 5(3)(1975), 453-474.

Benha University, Faculty of Science, Department of Mathematics, Benha 13518, Egypt. 